\title{
Expression of MICU1 after experimental focal cerebral ischemia in adult rats
}

\author{
Kun Zhang ${ }^{1,2}$, Jiajia Yan', Liang Wang ${ }^{1}$, Xinying Tian'1, Tong Zhang ${ }^{1}$, Bin Li², Wang Wang ${ }^{3}$, Li Guo ${ }^{1 *}$ \\ and Xiaoyun Liu ${ }^{1 *+}$
}

\begin{abstract}
Background: Mitochondrial $\mathrm{Ca}^{2+}$ uptake is a pivotal pathophysiological process for neuronal survival when subjected to ischemic insult. Mitochondrial calcium uptake 1 (MICU1) has been demonstrated as a key regulator of the mitochondrial calcium uniporter (MCU), identified as a tetrameric highly specific channel that modulates mitochondrial $\mathrm{Ca}^{2}+$ uptake.

Methods: Adult male Sprague-Dawley (SD) rats underwent middle cerebral artery occlusion (MCAO) to create the standard focal cerebral ischemia model. The permanent MCAO approach utilized the intraluminal approach. Neurological examination, and subsequent histological characterization of cerebral infarcts using triphenyltetrazolium chloride staining, as well as Western blot, immunohistochemical staining, and real-time quantitative polymerase chain reaction assays were employed to assess the functional effects of MICU1 and its expression in the brain.

Results: Animals exposed to MCAO displayed the typical neurological deficit accompanied by cortical and subcortical infarction at $72 \mathrm{~h}$ post-stroke. The expression of MICU1, with co-localization with neurons, was detected at different time points ( $6 \mathrm{~h}$ and $12 \mathrm{~h}$ ) after ischemic damage. Altogether, these observations revealed an up-regulation of MICU1 expression in the early stages of cerebral ischemia.

Conclusion: The results demonstrated that MICU1 was upregulated in neurons at the acute phase of ischemic stroke. Because MICU1 has been previously shown to participate in mitochondrial $\mathrm{Ca}^{2+}$ uptake mediated by MCU, our study further implicates the involvement of MICU1 in calcium overload-induced cell death which is closely associated with stroke.
\end{abstract}

Keywords: MICU1, Cerebral ischemia, MCU, $\mathrm{Ca}^{2+}$

\section{Background}

Cerebral ischemia-induced injury is a complex pathophysiological process. Although the primary cause of ischemic stroke has been attributed to blood flow interruption, the specific mechanisms underlying the progression of the disease, in particular the secondary cell death processes remain not fully understood. A widely postulated cell death pathway implicated in stroke involves mitochondrial $\mathrm{Ca}^{2+}$ overload induced by mitochondrial $\mathrm{Ca}^{2+}$ uptake. Such aberrant mitochondrial $\mathrm{Ca}^{2+}$ overload has been shown to play a vital role in the onset and progression of pathophysiological process in the early phases of ischemia [1]. Previous studies have

\footnotetext{
*Correspondence: guoli6@163.com; audrey-l@163.com

${ }^{\dagger}$ Equal contributors

${ }^{1}$ Department of Neurology, the Second Hospital of Hebei Medical University, No.215 West Heping Road, Shijiazhuang, Hebei Province 050000, China Full list of author information is available at the end of the article
}

demonstrated that the mitochondrial calcium uniporter (MCU), which possesses two transmembrane domains and forms tetrameric highly specific mitochondrial $\mathrm{Ca}^{2+}$ channels, is responsible for the mitochondrial $\mathrm{Ca}^{2+}$ uptake [2]. Mitochondrial $\mathrm{Ca}^{2+}$ uptake 1 (MICU1) has been demonstrated to be a key regulator of MCU [3]. This MICU1 regulation (albeit inhibition) of MCU activation occurs at low cytosolic free $\mathrm{Ca} 2+$ concentration $\left[\mathrm{Ca}^{2+}\right]_{\mathrm{c}}$, while MICU1 as an activator of MCU ensues at high $\left[\mathrm{Ca}^{2+}\right]_{\mathrm{c}}(2.5 \mu \mathrm{M})$ [3]. Interestingly, upregulated expression of $\mathrm{MCU}$ and increased $\left[\mathrm{Ca}^{2+}\right]_{\mathrm{c}}$ accompany the early stages of cerebral ischemic damage $[2,3]$.

In view of the reported interaction between MICU1 and $\mathrm{MCU}$, whereby MICU1 activates $\mathrm{MCU}$ at high $\left[\mathrm{Ca}^{2+}\right]_{\mathrm{C}}[3]$, we advanced the hypothesis that if indeed MICU1 is involved in the early pathogenesis of stroke, then MICU1 upregulation should be detected acutely in discrete regions 
of the ischemic stroke brain. Thus, we examined here the expression of MICU1 both at protein and mRNA levels at the acute stages of experimentally induced ischemic stroke in animals. The results from this study should provide insights into the specific timing and brain regions associated with MICU1 upregulation after cerebral ischemia.

\section{Methods}

\section{Animals and groups}

We used adult male Sprague-Dawley rats which weighed between 250 and $280 \mathrm{~g}$, purchased from Hebei Medical University Experimental Animal Center. All rats were kept on a $12 \mathrm{~h}$-light/12 h-dark with humidity of 55 $\pm 5 \%$, and had free access to food and water. Rats were randomly divided into eight groups as control (normal control), sham (sham operated), MCAO $3 \mathrm{~h}, \mathrm{MCAO}$ $6 \mathrm{~h}, \mathrm{MCAO} 12 \mathrm{~h}, \mathrm{MCAO} 24 \mathrm{~h}, \mathrm{MCAO} 48 \mathrm{~h}, \mathrm{MCAO}$ $72 \mathrm{~h}$. The brain samples were collected from the rats after decapitation. The sample size of $n=5$ was estimated based power analysis taking into account our previous studies demonstrating the minimum number of samples needed to demonstrate $25 \%$ difference between treatment groups.

\section{Permanent middle cerebral artery occlusion}

Rats underwent permanent middle cerebral artery occlusion (MCAO) as previously described $[4,5]$. After induction of anesthesia by intraperitoneal injection with $10 \%$ chloral hydrate $(250 \mathrm{mg} / \mathrm{kg})$, a nylon filament (diameter $0.265 \mathrm{~mm}$ ) was inserted intraluminally into the Internal carotid artery (ICA) about 18 to $20 \mathrm{~mm}$ deep. Gentamicin was used to protect against infection at the incision site. Rats of the sham group received the same surgery except for the filament insertion. The body temperatures of the rats were maintained at $37 \pm 1{ }^{\circ} \mathrm{C}$ by an electric blanket during the surgery. Moreover, physiological measures, including $\mathrm{pH}$, pCO2, pO2, and systolic/diastolic blood pressure were recorded, and did not significantly differ among animals, confirming a homogenous set of stroke animals was enrolled in this study.

\section{Neurological exam}

All investigators testing the animals were blinded to the treatment condition. Animals were subjected to neurological exam by using a scale for grading the ischemic injuryinduced dysfunctions as follows: 0 , rats extend straight both forelimbs, indicating no observable deficit; 1 , rats keep the left forelimb to the breast and extend straight the right forelimb; 2, rats show decreased resistance to lateral push in addition to behavior in score 1 without circling; 3 , rats twist the upper half of their body in addition to behavior in score 2. The animal was subjected to this test 3 times, and the scores from all 3 tests were averaged to give a mean neurologic deficit score (maximum possible score, 9 points divided by 3 tests $=3$ ).

\section{Cerebral infarction assay}

The volume of infarction was analyzed using the triphenyltetrazolium chloride (TTC), a histological assay for determining dehydrogenase activity. Under deep anesthesia (chloral hydrate, $500 \mathrm{mg} / \mathrm{kg}$, i.p.) animals were perfused intracardiacally with saline. Brains were then harvested, blocked, and processed for TTC staining. The volume of infarction was measured in each slice and summed using computerized planimetry (PC-based Image Tools software). The volume of infarction was computed as: $2 \mathrm{~mm}$ (thickness of the slice) $\times$ [sum of the infarction area in all brain slices $\left.\left(\mathrm{mm}^{2}\right)\right]$. To minimize artifacts produced by post-ischemic edema in the infarcted area, the infarction area in the ipsilateral hemisphere was indirectly measured by subtracting the non-infarcted area in the ipsilateral hemisphere from the total intact area of the contralateral hemisphere.

\section{Western blot}

The Total Protein Extraction Kit (Applygen Technologies Inc., Beijing, China) was used to obtain the total protein of the brain tissue, focusing on the peak area of ischemic tissue (bregma 1 to $-1 \mathrm{~mm}$ ). About $30 \mu \mathrm{g}$ total protein samples were applied to electrophoresis and then transferred onto PVDF membranes (Millipore Corporation, USA) as previously described [6]. After being blocked with $5 \%$ milk for $1 \mathrm{~h}$ at room temperature, the membranes were incubated overnight at $4{ }^{\circ} \mathrm{C}$ with antiMICU1 (1:500, Santa Cruz Biotechnology) diluted in 5\% milk. Polyclonal mouse anti-beta actin antibody (1:3000, Bioworld Technology) was used as a loading control. Membranes were incubated by fluorescent labeling second antibodies (IRDye 800-conjugate rabbit anti-goat IgG or rabbit anti-mouse IgG 1:5000 dilution; Rockland, Gilbertsville, PA, USA) for $1 \mathrm{~h}$ at room temperature. We analyzed the relative density of bands on the Odyssey infrared scanner (LICOR Bioscience, Lincoln, NE, USA).

\section{Immunohistochemical staining}

Paraffin-embedded sections were used to evaluate the expression of MICU1 at $6 \mathrm{~h}$ and $12 \mathrm{~h}$ after MCAO as previously described [7]. The brains of the rats were fixed in $4 \%$ paraformaldehyde over $24 \mathrm{~h}$ at $4{ }^{\circ} \mathrm{C}$, embedded in paraffin after being dehydrated in different gradients of alcohols. Then cut the brain into $5 \mu \mathrm{m}$ per slice. Brain sections were blocked in 3\% normal donkey serum and then incubated with MICU1 goat polyclonal antibody (1: 100, Santa Cruz Biotechnology) overnight at $4{ }^{\circ} \mathrm{C}$. The secondary antibodies were used the next day (Zhongshan Biology Technology Company, Beijing, China). Five visual fields of the ischemic regions (bregma 1 to $-1 \mathrm{~mm}$ which corresponded to core 
and peri-infarct areas to fully capture the extent of cell death) were selected to count the mean density value under a $400 \times$ microscope. A separate cohort of animals were also subjected to MCAO, with animals euthanized at $6 \mathrm{~h}$ and $12 \mathrm{~h}$ after stroke, and brains harvested as above, but processed for double-labeling of MICU1 (1: 100, Santa Cruz Biotechnology) and the neuronal marker MAP2 (1:100 Abcam). The regions of interest for estimation of MICU1 expression and MAP2 positive cells included the peak area of ischemic regions (bregma 1 to $-1 \mathrm{~mm}$ ), which were photographically captured (Axiophot2; Zeiss), and cells were quantified by counting per HPF view selected at random and corrected by the Abercrombie formula. Six captured fields in each coronal level, using three anterior-posterior levels, were used to analyze the MICU1 expression and MAP2 positive cells, using Scion Image software (Scion, Frederick, MD). Binary images were created using a distinct threshold, and then the positive areas were calculated.

\section{Real-time quantitative polymerase chain reaction}

Rats' brain tissue, also focusing on the peak area of ischemic tissue (bregma 1 to $-1 \mathrm{~mm}$ ), was rapidly dissected and the total RNA was extracted using Trizol (Invitrogen, Carlsbad, CA, USA). RNA (2 $\mu \mathrm{g})$ from each sample was applied to reverse transcribed for synthesized cDNA. Primers were synthesized by Shanghai Biological Engineering Technology Company Limited. Forward and reverse primers were 5'TTCCTCACAACGGTGCTCTC3' and 5'-CCAGACTTGAGGGTGTTCCC-3' for MICU1 and 5'-CCCATCTATGAGGGTTACGC-3' and 5'-TTTA ATGTCACGCACGATTTC-3' for $\beta$-actin. Each sample was measured in triplicate and all samples were normalized by $\beta$-actin. Relative gene expression was calculated with the $2^{-\Delta \Delta C T}$ method and then analyzed using logarithmic transformation.

\section{Data and statistical analysis}

All results are shown as mean \pm standard error. One-way ANOVA followed by Student-Newman-Keuls and LSD tests were applied for multiple comparisons. Mann-Whitney $U$ test was applied for neurological deficit score. The differences with $P<0.05$ were considered statistically significant.

\section{Results}

\section{MCAO induces typical neurological deficits and cerebral} infarcts

The present induction of experimental stroke, MCAO, in adult rats replicated the well-established focal cerebral ischemia model which led to a significant cortical and subcortical infarction (Fig. 1a) corresponding neurological deficits (Fig. 1b). Cerebral infarcts was evident in TTC staining, which showed about $45 \%$ of the ipsilateral hemisphere infarcted compared to the contralateral intact hemisphere (Fig. 1c). The neurological deficits using the

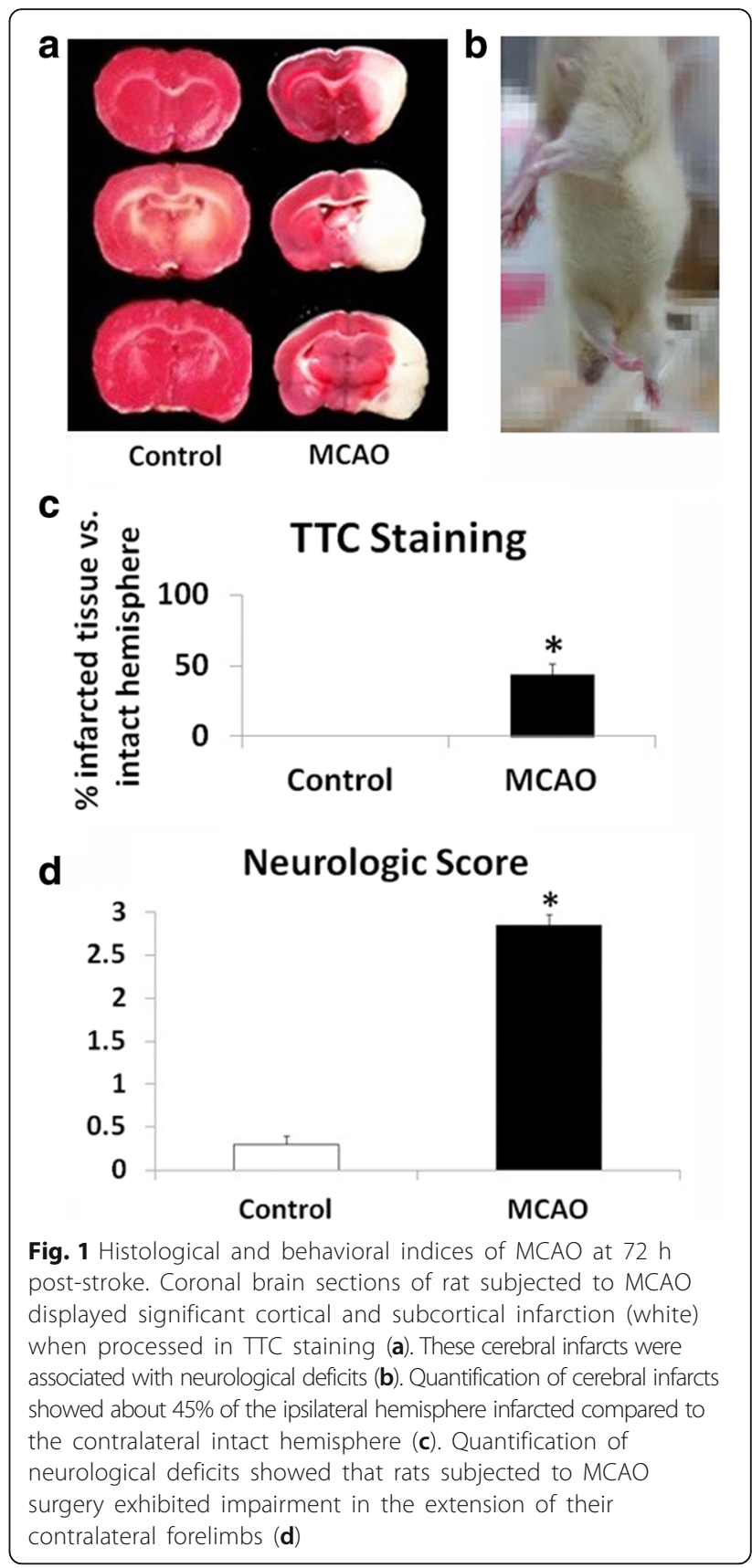

forelimb extension test confirmed that all rats subjected to MCAO surgery completely cannot extend the contralateral forelimb (Fig. 1d).

\section{MICU1 expression is significantly increased at protein level after cerebral ischemia}

We detected the expression of MICU1 at protein level by western blot with whole tissue lysates collected from the rats' brain regions from bregma 1 to $-1 \mathrm{~mm}$. Our results suggested that the expression of MICU1 was 


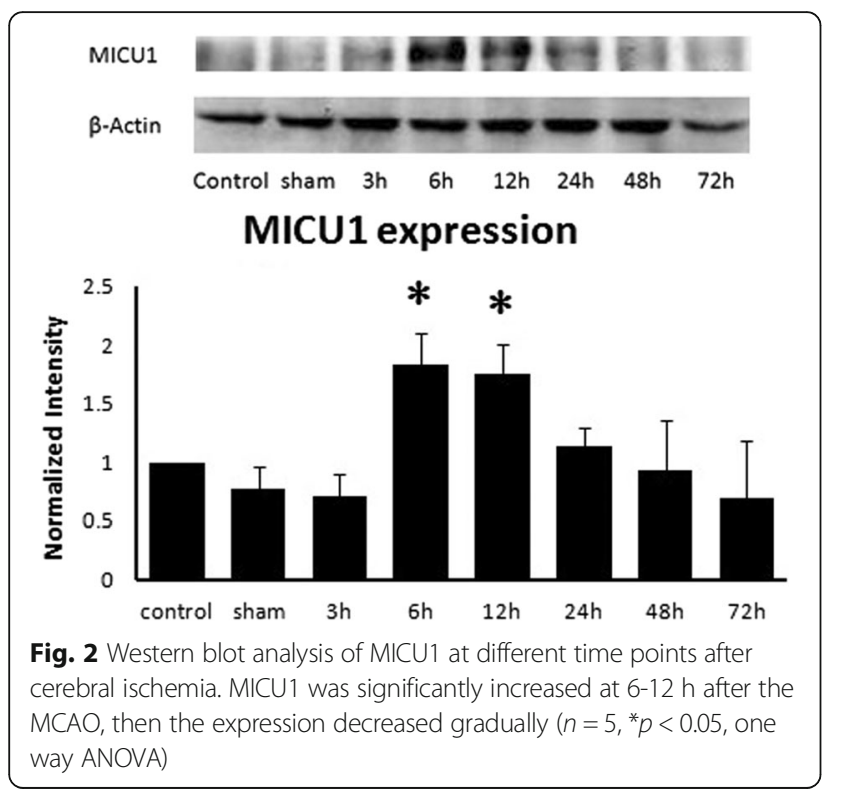

not notably changed at $3 \mathrm{~h}$ after ischemia, while significantly increased at 6-12 $\mathrm{h}$ after the middle cerebral artery occlusion, then the expression decreased gradually (Fig. 2).

We further measured the expression of MICU1 by immunohistochemistry in the neurons located in the same region of ischemic brains subject to MCAO at 6 and $12 \mathrm{~h}$ after ischemia. As expected, obviously increased MICU1 were found at both $6 \mathrm{~h}$ and12h (Fig. 3), which was consistent with the results of Western blot. These results suggested that the expression of MICU1 was indeed up-regulated at protein level after ischemia.

\section{Expression of MICU1 is significantly increased at mRNA level after cerebral ischemia}

In this study, we also measured the mRNA level of MICU1 at brain regions from bregma 1 to $-1 \mathrm{~mm}$ after cerebral ischemia. Real-time quantitative PCR (qPCR) was used to detect the change of MICU1 mRNA expression at different time points after MCAO in rats as previously described [8]. We obtained consistent results in Western blot assays, showing that mRNA expression of MICU1 increased at 6-12 h after brain ischemia, and gradually decreased after $12 \mathrm{~h}$ (Fig. 4).

\section{Discussion}

The present study revealed that MICU1 expression was upregulated in the early stage ( $6 \mathrm{~h}$ and $12 \mathrm{~h}$ ) of cerebral ischemia. The expression of MICU1 was co-localization with neurons in ischemic regions of the brain, suggesting an intimate association of MICU1 expression and the evolving pathology in acute stroke.

$\mathrm{Ca}^{2+}$ overload plays an important role in pathophysiological process after cerebral ischemic damage [1].
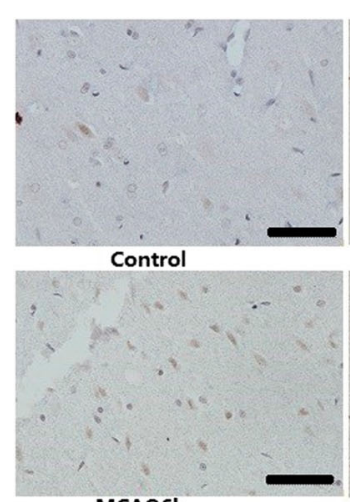

MCA06h

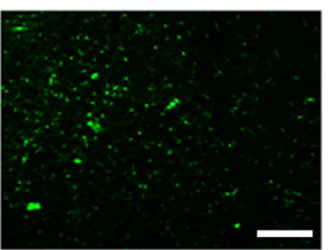

MICU1
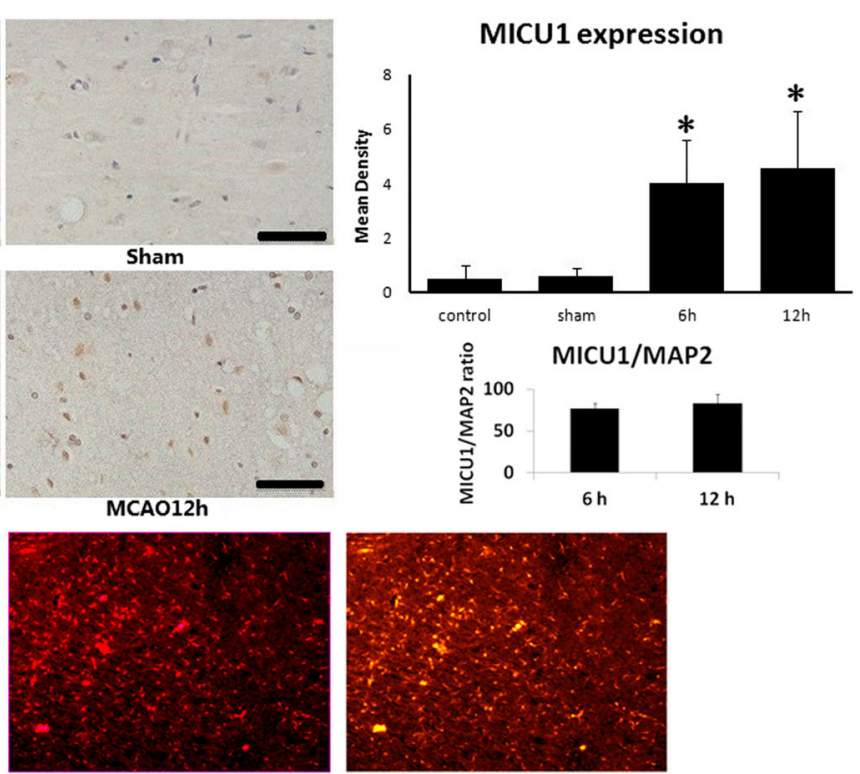

MAP2

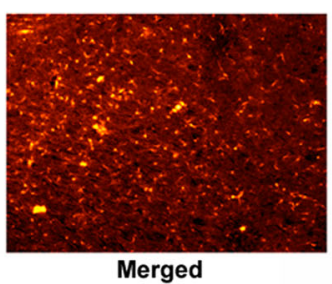

Fig. 3 Immunohistochemical staining of MICU1 at $6 \mathrm{~h}$ and $12 \mathrm{~h}$ after cerebral ischemia. Compared with control and sham groups, the mean density of MICU1 expression was significantly increased in both $6 \mathrm{~h}$ and $12 \mathrm{~h}$ after MCAO ( $n=5$, * $p<0.05$, one way ANOVA). Co-labeling of MICU1 and the neuronal marker MAP2 revealed about 80\% co-localization of phenotypic expression, as shown in MICU1/MAP2 ratio, in both 6 h and $12 \mathrm{~h}$ after MCAO. Scale bar $=100 \mu \mathrm{m}$ 


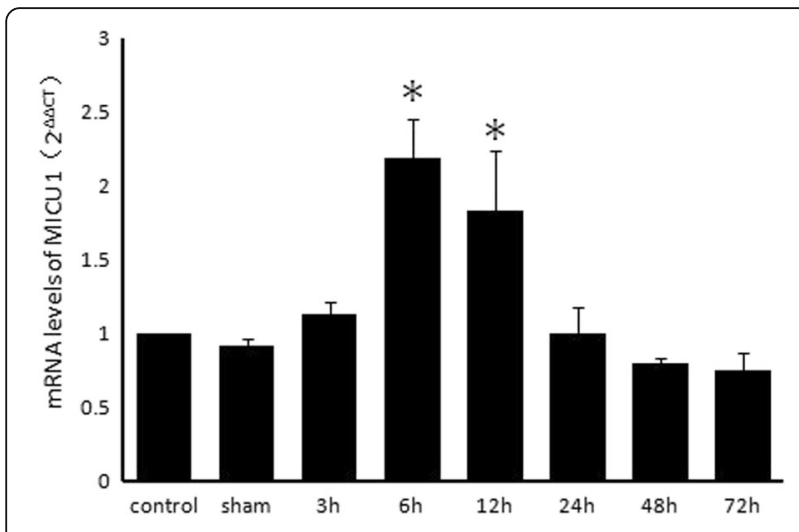

Fig. 4 Real-time quantitative PCR analysis of MICU1 at different time points after cerebral ischemia. Compared with normal control group and other time points, mRNA expression of MICU1 was increased at 6-12 $\mathrm{h}$ after MCAO ( $n=5,{ }^{*} p<0.05$, one way ANOVA)

Previous studies showed that a rise of calcium concentration signals an increased cellular energy demand and mediates a number of pathways that can precipitate mitochondrial injury and cell death [9]. Of interest, increments in $\left[\mathrm{Ca}^{2+}\right]_{\mathrm{c}}$ are detected at the acute phases after MCAO [10-12]. At approximately the same time, the expression of MCU is up-regulated [13]. Of interest, the aberrant pathophysiological cascade of cell death events at the early stage of cerebral ischemia may involve MCUmediated $\mathrm{Ca}^{2}+$ alterations that can lead to perturbed homeostasis $[1,9,13]$. That MICU1 is a key regulator of MCU implicates MICU1 serving a pivotal role in the initial progression of stroke. In particular, MICU1 can activate MCU at high $\left[\mathrm{Ca}^{2+}\right]_{\mathrm{c}}[3]$, which can subsequently promote MCU-induced mitochondrial $\mathrm{Ca}^{2+}$ uptake and ultimately cell death. However, this remains speculative as we have not yet established that the upregulation of MICU1 expression directly modulates MCAO onset and progression.

Additional studies are warranted to demonstrate causeand-effect relationship between MICU1 and MCAO, which may include the use gene and pharmacologic knockout or inhibition of MICU1. At best, our study showed that the expression of MICU1 was up-regulated in acute ischemic stroke, which paralleled previous studies demonstrating elevated $\left[\mathrm{Ca}^{2+}\right]_{\mathrm{c}}$ and increased MCU at the same time period.

\section{Conclusions}

Our results indicate that MICU1 upregulation accompanied acute ischemic stroke. Because MICU1 is involved in $\mathrm{Ca}^{2+}$ homeostasis by regulating MCU activation, this study supports MICU1 as a novel molecular target for abrogating the calcium overload detected in the early phase of cerebral ischemia.

\section{Abbreviations}

MCAO: Middle cerebral artery occlusion; MCU: Mitochondrial calcium uniporter; MICU1: Mitochondrial calcium uptake 1; qPCR: Real time quantitative PCR; SD: Sprague-Dawley

\section{Acknowledgement}

The authors acknowledge the funding sources listed above. The authors thank Dr. Cesario V Borlongan, of Department of Neurosurgery and Brain Repair and Center of Excellence for Aging and Brain Repair in University of South Florida College of Medicine, for his help and enlightening discussions with paper submission and revising.

\section{Funding}

This research was supported primarily by NSFC grants to Xiaoyun Liu from the National Natural Science Foundation of China (81571160); Second Hospital of Hebei Medical University Research Fund (2 h2201506); Key project of medical research in Hebei Province (20150668), and in part by Washington Institute for Health Sciences fund.

\section{Availability of data and materials}

The datasets supporting the conclusions of this article are available at the investigators' research centers located at the Second Hospital of Hebei Medical University.

\section{Authors' contributions}

The authors contributed equally to the conceptualization and write-up of this manuscript. All authors read and approved the final manuscript.

\section{Competing interests}

The authors declare that they have no competing interests.

\section{Consent for publication}

Not applicable.

\section{Ethics approval and consent to participate}

The use of animals in this study received approval from the Second Hospital of Hebei Medical University (Research Ethics Committee of the Second Hospital of Hebei Medical University, Permit Number: 2016131).

\section{Author details}

${ }^{1}$ Department of Neurology, the Second Hospital of Hebei Medical University, No.215 West Heping Road, Shijiazhuang, Hebei Province 050000, China. ${ }^{2}$ Department of Biochemistry and Molecular and Cellular Biology, Georgetown University, Georgetown Washington DC 20007, USA. ${ }^{3}$ Mitochondria and Metabolism Center, University of Washington, Seattle 98105, USA.

Received: 16 July 2016 Accepted: 11 February 2017

Published online: 02 June 2017

\section{References}

1. Starkov AA. The molecular identity of the mitochondrial Ca2+ sequestration system. FEBS J. 2010;277(18):3652-63.

2. Waldeck-Weiermair M, Malli R, Parichatikanond W, et al. Rearrangement of MICU1 multimers for activation of MCU is solely controlled by cytosolic Ca2 +. Sci Rep. 2015:5:15602

3. Matesanz-Isabel J, Arias-del-Val J, Alvarez-Illera P, et al. Functional roles of MICU1 and MICU2 in mitochondrial Ca2+ uptake. Biochimica et Biophysica Acta. 2016:1858(6);1110-17.

4. Longa EZ, Weinstein PR, Carlson S, Cummins R. Reversible middle cerebral artery occlusion without craniectomy in rats. Stroke. 1989;20(1):84-91.

5. Yang C, Zhang X, Fan H, Liu Y. Curcumin upregulates transcription factor $\mathrm{Nrf2,} \mathrm{HO}-1$ expression and protects rat brains against focal ischemia. Brain Res. 2009;1282:133-41.

6. Martel MA, Ryan TJ, Bell KF, et al. The subtype of GluN2 C-terminal domain determines the response to excitotoxic insults. Neuron. 2012;74:543-56.

7. Qiao HM, Dong LP, Zhang XJ, et al. Protective effect of luteolin in experimental ischemic stroke: upregulated SOD1, CAT, BCl-2 and claudin-5, down-regulated MDA and Bax expression. Neurochem Res. 2012;37:2014-24. 
8. Plengvidhya N, Chanprasert K, Tangjittipokin W, et al. Identification of copy number variation of CAPN10 in Thais with type 2 diabetes by multiplex PCR and denaturing high performance liquid chromatography (DHPLC). Gene. 2012:506:383-6.

9. Bhosale G, Sharpe JA, Sundier SY, et al. Calcium signaling as a mediator of cell energy demand and a trigger to cell death. Ann NY Acad Sci. 2015;1350:107-16.

10. Tang Z, Li S, Han P, Yin J, Gan Y, Liu Q, Wang J, Wang C, Li Y, Shi J. Pertussis toxin reduces calcium influx to protect ischemic stroke in a middle cerebral artery occlusion model. J Neurochem. 2015;135:998-1006.

11. Li LH, Tian XR, Hu ZP. The key target of neuroprotection after the onset of ischemic stroke: secretory pathway $\mathrm{Ca}(2+)$-ATPase 1. Neural Regen Res. 2015:10:1271-8

12. D'Orsi B, Kilbride SM, Chen G, et al. Bax regulates neuronal Ca2+ homeostasis. J Neurosci. 2015:35:1706-22.

13. Zhao Q, Wang S, Li Y, Wang P, Li S, Guo Y, Yao R. The role of the mitochondrial calcium uniporter in cerebral ischemia/reperfusion injury in rats involves regulation of mitochondrial energy metabolism. Mol Med Rep. 2013;7:1073-80

Submit your next manuscript to BioMed Central and we will help you at every step:

- We accept pre-submission inquiries

- Our selector tool helps you to find the most relevant journal

- We provide round the clock customer support

- Convenient online submission

- Thorough peer review

- Inclusion in PubMed and all major indexing services

- Maximum visibility for your research

Submit your manuscript at www.biomedcentral.com/submit
Biomed Central 